\title{
Jan M. Lundberg
}

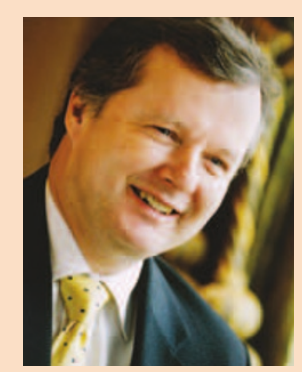

Jan M. Lundberg, Executive Vice-President, Head of Global Discovery Research, AstraZeneca

Jan Lundberg had medical training at the University of Gothenburg before obtaining his $\mathrm{PhD}$ and subsequently becoming professor in the Department of Pharmacology at the Karolinska Institute in Sweden, where he worked on cell signalling. He joined Astra $A B$ in 1995 as Head of Preclinical Affairs, and was then promoted to Senior Vice President, Head of Global Discovery, after the Astra-Zeneca merger in 1999, before taking on his current role as Head of Global Discovery Research in 2002. He has published more than 500 scientific articles in the cell signalling field, has been a member of the Swedish Medical Research Council and the Advisory Board for Drug Approvals at the Swedish Medical Product Agency, and in January 2003 was appointed Honorary Doctor of Pharmacy at Uppsala University.

AstraZeneca has had several setbacks with Crestor, Iressa, Exanta and now Galida. Is there a problem with drug development at $\mathrm{AZ}$ ?

There are risks associated with introducing new therapeutic paradigms like Iressa and Exanta. Since Discovery is contributing with, for example, translational science and safety assessment, we need to provide solutions together with our Development colleagues to satisfy regulators. However, I do feel that the regulators and the media need to appreciate that the issue is benefit versus risk and not only risk. As an industry we have a responsibility to patients to bring forward innovative treatments. The alternative is to forever stick with suboptimal and in some cases dangerous therapies, such as warfarin.

But with Iressa, isn't the issue with efficacy, not safety?

I'm certain that efficacy will be shown for Iressa in the correct patient population. A subpopulation of non-small-cell lung cancer patients are really benefiting from Iressa, possibly due to gain-of-function mutations in the EGFR pathway. In clinical practice, patients have been given Iressa and symptom improvements have been observed with treatment. Another way, although more invasive and time-consuming, would be to take tumour biopsies and analyse for mutations and make treatment decisions on that basis.

Are all of AZ's recent setbacks a reflection of ineffective trial design?

The paradigm for when critical decisions are made in drug development is changing and for novel drugs we're still learning how and when to choose the right sub-population. Ideally, you would have biomarkers or diagnostic tests when you start early clinical trials, but these tests are often only available once the drug has been conditionally approved and is on the market. I think our recent experiences will help us to design the optimal clinical trials from the beginning in future programmes.

That's quite an expensive experiment, isn't it? It is a key part of pharmaceutical R\&D to apply new advances in science and technology to overcome these challenges, which has been a characteristic of the pharmaceutical industry for many years.

How will recent setbacks affect your R\&D investment?

It has not affected R\&D investment. In times of difficulty it is pleasing that we had a record output from discovery and early development this year. Our expansion of drug discovery and efforts to increase productivity since the merger are really paying off.

If all four drugs above don't make it, what's in the pipeline and what changes to development will you make?

We are committed to innovative pharmaceutical research and the development of new medicines that benefit patients and improve health. We have several upcoming Phase III opportunities and encouraging recent data for Cerovive in stroke, but will certainly continue to work hard on the projects we have had difficulties with to turn them into successes. For example, Prilosec was considered problematic by regulatory authorities and then became the world's largest selling pharmaceutical, so we have not seen the end of our novel agents. Discovery of new therapies is a long-term activity and experience tells us that the most successful discovery organizations in big pharma are those that have a combination of pressure of delivery but also a long-term vision and persistence.

\section{Is $\mathrm{AZ}$ going to focus more on targeted} therapies now?

Discovery will continue to be opportunitydriven and depend on our scientists' ideas and how well these comply with the target-product profiles created by medical need and market demand. I agree that more targeted approaches can get us faster to market and then, after approval, the indications can be expanded.

What is your response to suggestions that AZ marketed Crestor too quickly because of financial difficulties?

We are an ethical company that introduces our products after regulatory agency approval based on their safety and efficacy for patients, and Crestor is now approved in 73 countries worldwide.

How much responsibility do you think the regulators should take for drug safety? They clearly have a key role and I think the pharmaceutical industry and regulators should have a partnership, together with society, to produce better and safer medicines.

And when those within the partnership give conflicting views?

Judgement should be made on the overall risk/ benefit related to the current available therapy and severity of the disease.

Within the FDA alone there have been conflicting views about Crestor. How do you feel about that?

We have been very public about the properties of Crestor and the data are available on a dedicated website. We have very good clinical evidence showing that Crestor is more efficacious with a similar safety profule compared with other marketed statins. 